\title{
Stability behavior of second order neutral impulsive stochastic differential equations with delay
}

\author{
S. Karunanithi \\ Department of Mathematics, \\ Kongunadu Arts and Science College (Autonomous), \\ Coimbatore - 641 029, Tamilnadu, India
}

\author{
R .Maheswari \\ Department of Mathematics, \\ Sri Eshwar College of Engineering, \\ Coimbatore - 642 109, Tamilnadu, India
}

\begin{abstract}
In this article, we study the existence and asymptotic stability in pth moment of mild solutions to second order neutral stochastic partial differential equations with delay. Our method of investigating the stability of solutions is based on fixed point theorem and Lipchitz conditions being imposed.

Keywords: Stochastic, neutral, impulse, asymptotic stability, mild solution
\end{abstract}

\section{INTRODUCTION}

Stochastic partial differential equations have received much attention in many areas of science including physics, biology, medicine and engineering. The existence, uniqueness and asymptotic behavior of solutions of the first order stochastic partial differential equations have been considered by several authors $[3,4,11,12,15,26]$. Moreover many dynamical systems not only depend on present and past states but also involve derivatives with delays. Deterministic neutral functional differential equations, which was originally introduced by Hale and Lunel [9], are of great interest in theoretical and practical applications. Kolmanovskii and Myshkis [13] introduced neutral stochastic functional differential equations and gave its applications in chemical engineering and aero elasticity considering environmental disturbances into account.

Caraballo et al. [5] have considered the exponential stability of neutral stochastic delay partial differential equations by the Lyapunov functional approach. In [7], Dauer and Mahmudov have analyzed the existence of mild solutions to semilinear neutral evolutions with nonlocal conditions by using the fractional power of operators and KransnoselskiSchaefer type fixed point theorem. In [10], Hu and Ren have established the existence results for impulsive neutral stochastic functional integrodifferential equations with infinite delays. It is well known that classical technique applied in the study of stability is based on a stochastic version of the Lyapunov direct method. However the Lyapunov direct method has some difficulty with the theory and application to specific problems when discussing the asymptotic behaviour of solutions in stochastic differential equations [16]. It seems that new methods are required to address those difficulties.

Appleby [1] studied the almost sure stability of stochastic differential equations with fixed point approach. Luo $[18,17]$ have successfully applied fixed point principle to investigate the stability of mild solution of various stochastic equations. Luo and Taniguchi [19], have studied the asymptotic stability of neutral stochastic partial differential equations with infinite delay by using the fixed point theorem. The impulsive effects exists widely in many evolution processes in which states are changed abruptly of certain moments of time, involving such fields as finance, economics, mechanics, electronics and telecommunications, etc [25]. The theory of impulsive differential equations have been studied extensively in $[21,22]$. However in addition to impulsive effects, stochastic effects likewise exist in real systems. It is well known that a lot of dynamical systems have variable structures subjects to stochastic abrupt changes, which may result from abrupt phenomena such as stochastic failures and repairs of the components, changes in the interconnections of subsystems, sudden environment changes, etc.

Even though there are many valuable results about neutral stochastic partial differential equations, they are mainly concerned with first-order case. In many cases it is advantageous to treat the second order stochastic differential equations rather than to convert them to first-order systems. The second-order stochastic differential equations are the right model in continuous time to account for integrated processes than can be made stationary. For instance, it is useful for engineers to model mechanical vibrations or charge on a capacitor or condenser subjected to white noise excitation through a second-order stochastic differential equations. The studies of the qualitative properties about abstract deterministic second order evolution equation governed by the generator of a strongly continuous cosine family was proposed in [8,27]. Mahmudov and McKibben [20] established results concerning the global existence and approximate controllability of mild solutions for a class of second order stochastic evolution equations. Moreover, Ren and Sun [23] established the existence, uniqueness and stability of the second-order neutral impulsive stochastic evolution equations with delay with some non-Lipschitz conditions. Balasubramaniam and Muthukumar [2] also discussed the approximate controllability of second-order neutral stochastic distributed implicit functional differential equations with infinite delay. Sakthivel et al. [24] have studied the asymptotic stability of second-order neutral stochastic differential equations by a fixed point theorem. Lei Zhang et al. [14] have studied the controllability of secondorder semilinear impulsive stochastic neutral functional evolution equations. Inspired by this consideration, the main objective of this paper is to study the asymptotic stability of the second-order neutral impulsive stochastic delay differential equations.

\section{PRELIMINARIES}

In this section, we briefly give some basic definitions and results for stochastic equations in infinite dimensions and cosine families of operators. We refer to Prato and Zabczyk [6] and Fattorini [8] for more details. Let $X$ and $E$ be two real separable Hilbert spaces and $L(E, X)$ be the space of bounded linear operators from $E$ into $X$, equipped with the usual operator norm $\|\cdot\|$. Let $(\Omega, \Gamma, P)$ be a complete probability space furnished with a normal filtration $\left\{\Gamma_{t}\right\}_{t \geq 0}$ generated by the $Q$-Wiener process $w$ 
on $(\Omega, \Gamma, P)$ with the linear bounded covariance operator $Q$ such that $\operatorname{tr} Q<\infty$. We assume that there exist a complete orthonormal system $\left\{e_{i}\right\}_{i \geq 1}$ in E, a bounded sequence of nonnegative real numbers $\left\{\lambda_{i}\right\}$ such that $Q e_{i}=\lambda_{i} e_{i}, i=1,2,3, \ldots, \quad$ and $\quad$ a sequence $\left\{\beta_{i}\right\}$ of independent Brownian motions such that $\langle w(t), e\rangle=\sum_{i=1}^{\infty} \sqrt{\lambda_{i}}\left\langle e_{i}, e\right\rangle \beta_{i}(t), e \in E$

and $\Gamma_{t}=\Gamma_{t}{ }^{w}$,where $\Gamma_{t}{ }^{w}$ is the sigma algebra generated by $\{\mathrm{w}(\mathrm{s}) ; \mathrm{t} \geq 0\}$. Let $L_{2}{ }^{0}=L_{2}\left(Q^{1 / 2} E ; X\right)$ denote the space of all Hilbert -Schmidt operators from $Q^{1 / 2} E$ to $X$ with the inner product $\langle\psi, \varphi\rangle_{L_{2}{ }^{0}}=\operatorname{tr}\left[\psi Q \varphi^{*}\right]$. Let $L^{p}\left(\Omega, \Gamma_{t}, X\right)$ is the Hilbert space of all $\Gamma_{t}$-measurable square integrable random variables with values in a Hilbert space $X$.

In this paper, we consider the following second-order neutral impulsive stochastic differential equations with delays of the form

$$
\begin{aligned}
& d\left[x^{\prime}(t)-f_{0}(t, x(t-\delta(t)))\right]=\left[A x(t)+f_{1}(t, x(t-\rho(t)))\right] d t \\
& +f_{2}(t, x(t-\sigma(t))) d \omega(t), t \geq 0 \\
& x_{0}(\cdot)=\varphi \in D_{\Gamma_{0}}^{b} ; \quad x^{\prime}(0)=x_{1} \\
& \Delta x\left(t_{k}\right)=I_{k}\left(x\left(t_{k}\right)\right) \Delta x^{\prime}\left(t_{k}\right)=\widetilde{I}_{k}\left(x\left(t_{k}\right)\right)
\end{aligned}
$$

$$
\text { where } k=1,2, \ldots, m \text {, }
$$

where $\varphi \in D_{\Gamma_{0}}^{b}$ and $x_{1}$ is also an $\Gamma_{0}$-measurable $X$. valued random variables independent of $w$. $A: D(A) \subset X \rightarrow X \quad$ is the infinitesimal generator of a strongly continuous cosine family on $X$; $f_{i}: R_{+} \times X \rightarrow X(i=0,1) ; f_{2}: R_{+} \times X \rightarrow L_{2}^{0}$ are appropriate mappings and $I_{k}, \widetilde{I}_{k}: X \rightarrow X$ are appropriate functions. Moreover, let

$0=t_{0}<t_{1}<\ldots<t_{m}<t_{m+1}=\infty$,

$\Delta x\left(t_{k}\right)=x\left(t_{k}^{+}\right)-x\left(t_{k}^{-}\right)$,

$\Delta x^{\prime}\left(t_{k}\right)=x^{\prime}\left(t_{k}^{+}\right)-x^{\prime}\left(t_{k}^{-}\right), x\left(t_{k}^{+}\right)$and $x\left(t_{k}^{-}\right)$ denote the right and left limits of $x$ at $t_{k}$. Similarly $x^{\prime}\left(t_{k}{ }^{+}\right)$ and $x^{\prime}\left(t_{k}^{-}\right)$denote the right and left limits of $x^{\prime}$ at $t_{k}$. Moreover $I_{k}, \widetilde{I}_{k}$ represents the size of the jump. Let $\delta, \rho, \sigma: R_{+} \rightarrow[0, \tau](\tau>0)$ are continuous. The space $\mathrm{D}$ is assumed to be equipped with the norm
$\|\varphi\|_{D}=\sup _{-\tau \leq t \leq 0}\|\varphi(t)\|_{X}$. Here $D_{\Gamma_{0}}^{b}([-\tau, 0], X)$ denote the family of all almost surely bounded, $\Gamma_{0}$ measurable, continuous random variables from $[-\tau, 0]$ to $X$. Let us introduce the spaces

$$
\begin{aligned}
& H([0, T] ; X)=\left\{x: J \rightarrow X,\left.x\right|_{\left(t_{k}, t_{k+1]}\right.} \in C\left(\left(t_{k}, t_{k+1}\right], X\right),\right. \\
& k=1,2,3, \ldots, m \\
& \text { and there exist } \left.x\left(t_{k}^{+}\right) \text {for } k=1,2,3, \ldots, m\right\} \text { and } \\
& H^{\prime}([0, T] ; X)=\left\{x \in H([0, T] ; X),\left.x\right|_{\left(t_{k}, t_{k+1}\right.} \in\right. \\
& \qquad C^{\prime}\left(\left(t_{k}, t_{k+1}\right], X\right), k=1,2,3, \ldots, m
\end{aligned}
$$

and there exist $x^{\prime}\left(t_{k}{ }^{+}\right)$for $\left.k=1,2,3, \ldots, m\right\}$.

It is obvious that $H([0, T] ; X)$ and $H^{\prime}([0, T] ; X)$ are Banach spaces endowed with the norm $\|x\|_{H}=\sup _{t \in[0, T]} E\|x(t)\|_{X}^{p}$.It is easy to see that $H^{\prime}$ provided with the norm $\|x\|_{H^{\prime}}=\|x\|_{H}+\left\|x^{\prime}\right\|_{H}$.

In this section, we mention some basic concepts, notations, and properties about cosine families of operators [8, 27].

Let $L(E ; X)$ is the space of bounded linear operators from $\mathrm{E}$ into $\mathrm{X}$. The one parameter family $\{C(t) ; t \in R\} \subset L(X)$ satisfying

(i) $C(0)=I$,

(ii) $C(t) x$ is continuous in $t$ on $R$ for all $x \in X$,

(iii) $C(t+s)-C(t-s)=2 C(t) C(s)$

for all $t, s \in R$ is called a strongly continuous cosine family.

The corresponding strongly continuous sine family

$\{S(t) ; t \in R\} \subset L(X)$ is define

$S(t) x=\int_{0}^{t} C(s) x d s, t \in R, x \in X$.

The generator $A: X \rightarrow X$ of $\{C(t) ; t \in R\}$ is given by

$$
A x=\left.\left(d^{2} / d t^{2}\right) C(t) x\right|_{t=0} \text { for all }
$$$$
x \in D(A)=\left\{x \in X: C(\cdot) x \in C^{2}(R ; X)\right\}
$$

It is well known that the infinitesimal generator $A$ is a closed, densely defined operator on $X$. Such cosine and sine families and their generators satisfy the following properties.

\section{Lemma 2.1: [8]}

Suppose that $A$ is the infinitesimal generator of a cosine family of operators $\{C(t) ; t \in R\}$. Then the following terms hold. 
(i) There exists $M^{*} \geq 1$ and $\alpha \geq 0$ such that $\|C(t)\| \leq M^{*} e^{\alpha|t|}$ and hence $\|S(t)\| \leq M^{*} e^{\alpha|t|}$.

(ii) $A \int_{s}^{\hat{r}} S(u) x d u=[C(\hat{r})-C(s)] x$ for all $0 \leq s \leq \hat{r} \leq \infty$.

(iii) There exists $N^{*} \geq 1$ such that $\|S(s)-S(\hat{r})\| \leq N^{*}\left|\int_{s}^{\hat{r}} e^{\alpha|s|} d s\right|$ for all $0 \leq s \leq \hat{r} \leq \infty$.

Lemma 2.2: [6]. For any $r \geq 1$ and for arbitrary $L_{2}{ }^{0}$ - valued predictable process $\phi(\cdot)$ such that

$$
\sup _{s \in[0, t]} E\left\|\int_{0}^{s} \phi(u) d u(u)\right\|_{X}^{2 r} \leq(r(2 r-1))^{r}\left(\int_{0}^{t}\left(E\|\phi(s)\|_{L_{2}^{0}}^{2 r}\right)^{\frac{1}{r}} d s\right)^{r} .
$$

Definition 2.3: A stochastic process $\{x(t), t \in[0, T]\}$ $0 \leq T<\infty$ is called a mild solution of equations (1), (2) and (3) if

(i) $x(t)$ is adapted to $\Gamma_{t}, t \geq 0$.

(ii) $x(t) \in X$ had ca'dla'g paths on $t \in[0, T]$ a.s and for each $t \in[0, T], x(t)$ satisfies the integral equation

$$
\begin{aligned}
x(t)= & C(t) \varphi(0)+S(t)\left(x_{1}-f_{0}(0, x(0,-\delta(0)))\right) \\
& +\int_{0}^{t} C(t-s) f_{0}(s, x(s-\delta(s))) d s \\
& +\int_{0}^{t} S(t-s) f_{1}(s, x(s-\rho(s))) d s \\
& +\int_{0}^{t} S(t-s) f_{2}(s, x(s-\sigma(s))) d w(s) \\
& +\sum_{0<t_{k}<t} C\left(t-t_{k}\right) I_{k}\left(x\left(t_{k}\right)\right) \\
& +\sum_{0<t_{k}<t} S\left(t-t_{k}\right) \widetilde{I}_{k}\left(x\left(t_{k}\right)\right)
\end{aligned}
$$

Definition 2.4: Let $p \geq 2$ be an integer. Equation (3) is said to be asymptotically stable in pth moment if it is stable in pth moment and for any $\varphi \in D_{\Gamma_{0}}^{b}, x_{1} \in X$, we have

$$
\lim _{T \rightarrow \infty} E\left\{\sup \|x(t)\|_{X}^{p}\right\}=0
$$

\section{ASYMPTOTIC STABILITY OF SECOND-ORDER NEUTRAL STOCHASTIC DIFFERENTIAL EQUATIONS}

Now let us present the main result of this paper. We consider the asymptotic stability in the pth moment of mild solutions (1), (2), (3) by using the fixed point principle. Moreover, for the purpose of asymptotic stability, we shall assume that in this work $\mathrm{f}_{\mathrm{i}}(t, 0)=0(i=0,1)$ and $\mathrm{f}_{2}(t, 0)=0, \quad I_{k}(0)=0, \widetilde{I}_{k}(0)=0, k=1,2, \ldots, m$. Then equations (1), (2) and (3) have a trivial solution when $\varphi=0$ and $x_{1}=0$.

To prove the following result, we impose the following conditions.

(I) The cosine family of operators $\{C(t) ; t \geq 0\}$ on $\mathrm{X}$ and

the corresponding sine family $\{S(t) ; t \geq 0\}$ satisfy the conditions $\|C(t)\|_{X} \leq M e^{-b t}$,

$$
\|S(t)\|_{X} \leq M e^{-a t}, t \geq 0 \text { for some constants }
$$$$
M \geq 1 \text { and } 0<a, b \in R_{+} \text {. }
$$

(II) The functions $\mathrm{f}_{\mathrm{i}}(i=0,1,2)$ satisfy the Lipchitz condition and there exist positive constants $K_{1}, K_{2}, K_{3}$ for every $t \geq 0$ and $x, y \in X$, such that

$$
\begin{gathered}
\left\|f_{i}(t, x)-f_{i}(t, y)\right\|_{X} \leq K_{i}\|x-y\|_{X} ; i=0,1 . \\
\left\|f_{2}(t, x)-f_{2}(t, y)\right\|_{X} \leq K_{3}\|x-y\|_{X} ; i=2
\end{gathered}
$$

(III) The function $I_{k}, \widetilde{I}_{k}$ and there are positive constants $\mathrm{q}_{\mathrm{k}}, \mathrm{g}_{\mathrm{k}}$ such that

$$
\begin{aligned}
& \left\|I_{k}(x)-I_{k}(y)\right\|^{p} \leq q_{k}\|x-y\|^{p}, \\
& \left\|\widetilde{I}_{k}(x)-\widetilde{I}_{k}(y)\right\|^{p} \leq g_{k}\|x-y\|^{p},
\end{aligned}
$$

for each $x, y \in X \quad(k=1,2,3, \ldots, m)$.

(IV) $I_{k}(0)=0, \widetilde{I}_{k}(0)=0, k=1,2,3, \ldots, m$.

\section{Theorem 3.1:}

Assume the conditions (I)-(IV) hold. Let $p \geq 2$ be an integer and $c_{p}=\left(\frac{p(p-1)}{2}\right)^{\frac{p}{2}}$. If the inequality

$\left[5^{\mathrm{p}-1} \mathrm{M}^{\mathrm{p}}\left(\mathrm{k}_{1}{ }^{\mathrm{p}} \mathrm{b}^{-\mathrm{p}}+\mathrm{k}_{2}{ }^{\mathrm{p}} \mathrm{a}^{-\mathrm{p}}+\mathrm{k}_{3}{ }^{\mathrm{p}}(2 a)^{-\frac{p}{2}}+\hat{L}+\hat{D}\right)\right]<1$ is satisfied, then the second-order neutral stochastic differential equations with delays (1), (2) and (3) is asymptotically stable in pth moment.

Proof: Define an operator $\Psi: H \rightarrow H$ by 


$$
\begin{aligned}
& \Psi(x)(t)=C(t) \varphi(0)+S(t)\left(x_{1}-f_{0}(0, x(0,-\delta(0)))\right) \\
& \quad+\int_{0}^{t} C(t-s) f_{0}(s, x(s-\delta(s))) d s \\
& +\int_{0}^{t} S(t-s) f_{1}(s, x(s-\rho(s))) d s \\
& +\int_{0}^{t} S(t-s) f_{2}(s, x(s-\sigma(s))) d w(s) \\
& +\sum_{0<t_{k}<t} C\left(t-t_{k}\right) I_{k}\left(x\left(t_{k}\right)\right)+\sum_{0<t_{k}<t} S\left(t-t_{k}\right) \widetilde{I}_{k}\left(x\left(t_{k}\right)\right) \\
& =\sum_{i=1}^{7} F_{i}(t) ; t \geq 0 .
\end{aligned}
$$

In order to prove the asymptotic stability, it is enough to prove that the operator $\Psi$ has a fixed point $H$.To prove this result, we use the contraction mapping principle. To apply the contraction mapping principle, first we verify the mean square continuity of $\Psi$ on $[0, \infty)$.

Let $x \in H, t_{1} \geq 0$ and $|r|$ is sufficiently small then

$$
\begin{aligned}
E\left\|\Psi(x)\left(t_{1}+r\right)-\psi(x)\left(t_{1}\right)\right\|_{X}^{p} & \\
& \leq 7^{p-1} \sum_{i=1}^{7} E\left\|F_{i}\left(t_{1}+r\right)-F_{i}\left(t_{1}\right)\right\|_{X}^{p} .
\end{aligned}
$$

We can see that

$$
E\left\|F_{i}\left(t_{1}+r\right)-F_{i}\left(t_{1}\right)\right\|_{X}^{p} \rightarrow 0, i=1,2,3,4,6,7 \text { as } r \rightarrow 0 \text {. }
$$

Moreover by using Holder's inequality and lemma 2.2, we obtain

$$
\begin{gathered}
E\left\|F_{5}\left(t_{1}+r\right)-F_{5}\left(t_{1}\right)\right\|_{X}^{p} \\
\leq 2^{p-1} c_{p}\left[\int_{0}^{t_{1}}\left(E \|\left(\begin{array}{r}
\left.\left(t_{1}+r-s\right)-S\left(t_{1}-s\right)\right) \\
\times f_{2}(s, x(s-\sigma(s)))
\end{array} \|_{X}^{p}\right)^{(2 / p)} d s\right]^{(p / 2)}\right. \\
+2^{p-1} c_{p}\left[\int_{t_{1}}^{t_{1}+r}\left(E\left\|S\left(t_{1}+r-s\right) f_{2}(s, x(s-\sigma(s)))\right\|_{X}^{p}\right)^{(2 / p)} d s\right]^{(p / 2)} \\
\rightarrow 0 \text { as } r \rightarrow 0,
\end{gathered}
$$

Thus, $\Psi$ is continuous in pth moment on $[0, \infty)$.

Next, we show that $\Psi(H) \subset H$. From (5), we obtain

$$
\begin{aligned}
& E\|(\psi x)(t)\|_{x}^{p} \leq 8^{p-1} E\|C(t) \varphi(0)\|_{X}^{p} \\
& +8^{p-1} E\left\|S(t) x_{1}\right\|_{X}^{p}+8^{p-1} E\left\|S(t) f_{0}(0, x(0-\delta(0)))\right\|_{X}^{p} \\
& +8^{p-1} E\left\|\int_{0}^{t} C(t-s) f_{0}(s, x(s-\delta(s))) d s\right\|_{X}^{p} \\
& +8^{p-1} E\left\|\int_{0}^{t} S(t-s) f_{1}(s, x(s-\rho(s))) d s\right\|_{X}^{p} \\
& +8^{p-1} E\left\|\int_{0}^{t} S(t-s) f_{2}(s, x(s-\sigma(s))) d w(s)\right\|_{X}^{p} \\
& +8^{p-1} \sum_{0<t_{k}<t} E\left\|C\left(t-t_{k}\right) I_{k}\left(x\left(t_{k}\right)\right)\right\|_{X}^{p} \\
& +8^{p-1} \sum_{0<t_{k}<t} E\left\|S\left(t-t_{k}\right) \widetilde{I}_{k}\left(x\left(t_{k}\right)\right)\right\|_{X}^{p} .
\end{aligned}
$$

Now, we estimate the terms on the right hand side of (7) using ( $I),(I I),(I I I)$ and (IV) we obtain

$$
\begin{aligned}
& 8^{p-1} E\|C(t) \varphi(0)\|_{X}^{p} \\
& \leq 8^{p-1} M^{p} e^{-b p t}\|\varphi\|_{D}^{p} \rightarrow 0 \quad \text { as } t \rightarrow \infty \\
& 8^{p-1} E\left\|S(t) x_{1}\right\|_{X}^{p} \\
& \leq 8^{p-1} M^{p} e^{-a p t}\left\|x_{1}\right\|_{X}^{p} \rightarrow 0 \quad \text { as } t \rightarrow \infty \\
& 8^{p-1} E\left\|S(t) f_{0}(0, x(0,-\delta(0)))\right\|_{X}^{p} \\
& \leq 8^{p-1} M^{p} e^{-a p t} K_{1}\|x(-\delta(0))\|_{X}^{p} \rightarrow 0 \text { as } t \rightarrow \infty,(10) \\
& 8^{p-1} \sum_{0<t_{k}<t} E\left\|C\left(t-t_{k}\right) I_{k}\left(x\left(t_{k}\right)\right)\right\|_{X}^{p} \\
& \leq 8^{p-1} M^{p} e^{-p b t}\left\|I_{k}\left(x\left(t_{k}\right)\right)\right\|_{X}^{p} \rightarrow 0 \text { as } t \rightarrow \infty,(11) \\
& 8^{p-1} \sum_{0<t_{k}<t} E\left\|S\left(t-t_{k}\right) \widetilde{I}_{k}\left(x\left(t_{k}\right)\right)\right\|_{X}^{p} \\
& \leq 8^{p-1} M^{p} e^{-p a t}\left\|\widetilde{I}_{k}\left(x\left(t_{k}\right)\right)\right\|_{X}^{p} \rightarrow 0 \text { as } t \rightarrow \infty,(12)
\end{aligned}
$$

From I, II, III, (IV) and Holder's inequality, we have

$$
\begin{aligned}
& 8^{p-1} E\left\|\int_{0}^{t} C(t-s) f_{0}(s, x(s-\delta(s))) d s\right\|_{X}^{p} \\
& \leq 8^{p-1} M^{p} K_{1}^{p}\left[\int_{0}^{t} e^{-b(t-s)} d s\right]_{0}^{p-1} \int_{0}^{t} e^{-b(t-s)} E\|x(s-\delta(s))\|_{X}^{p} d s \\
& \leq 8^{p-1} M^{p} K_{1}^{p} b^{1-p} \int_{0}^{t} e^{-b(t-s)} E\|x(s-\delta(s))\|_{X}^{p} d s .
\end{aligned}
$$


For any $x(t) \in H$ and any $\varepsilon>0$, there exist a $t_{1}>0$, such that $E\|x(s-\delta(s))\|_{X}^{p}<\varepsilon$ for $t \geq t_{1}$.

Thus from (13), we obtain

$$
\begin{gathered}
8^{p-1} E\left\|\int_{0}^{t} C(t-s) f_{0}(s, x(s-\delta(s))) d s\right\|_{X}^{p} \\
\leq 8^{p-1} M^{p} K_{1}^{p} b^{1-p} e^{-b t} \int_{0}^{t_{1}} e^{b s} E\|x(s-\delta(s))\|_{X}^{p} d s \\
+8^{p-1} M^{p} K_{1}^{p} b^{-p} \varepsilon .
\end{gathered}
$$

As $e^{-b t} \rightarrow 0$ as $t \rightarrow \infty$ and by assumption on Theorem 3.1 , there exists $t_{2}>t_{1}$, such that for any $t \geq t_{2}$, we have

$$
\begin{gathered}
8^{p-1} M^{p} K_{1}^{p} b^{1-p} e^{-b t} \int_{0}^{t_{1}} e^{b s} E\|x(s-\delta(s))\|_{X}^{p} d s \\
\leq \varepsilon-8^{p-1} M^{p} K_{1}^{p} b^{-p} \varepsilon
\end{gathered}
$$

From (14) and (15), for any $t \geq t_{2}$, we obtain

$$
8^{p-1} E\left\|\int_{0}^{t} C(t-s) f_{0}(s, x(s-\delta(s))) d s\right\|_{X}^{p}<\varepsilon .
$$

That is to say,

$$
\begin{array}{r}
8^{p-1} E\left\|\int_{0}^{t} C(t-s) f_{0}(s, x(s-\delta(s))) d s\right\|_{X}^{p} \rightarrow 0 \\
\text { as } t \rightarrow \infty \text {. (16) }
\end{array}
$$

Similarly we can obtain

$$
\begin{array}{r}
8^{p-1} E\left\|\int_{0}^{t} S(t-s) f_{1}(s, x(s-\rho(s))) d s\right\|_{X}^{p} \rightarrow 0 \\
\text { as } t \rightarrow \infty \text {. (17) }
\end{array}
$$

Now for any $x(t) \in S, t \in[-\tau, \infty]$, we have

$$
\begin{aligned}
& 8^{p-1} E\left\|\int_{0}^{t} S(t-s) f_{2}(s, x(s-\sigma(s))) d \omega(s)\right\|_{X}^{p} \\
& \leq 8^{p-1} c_{p} M^{p}\left[\int_{0}^{t}\left(e^{-a p(t-s)} E\left\|f_{2}(s, x(s-\sigma(s)))\right\|_{X}^{p}\right)^{(2 / p)} d s\right]^{(p / 2)}
\end{aligned}
$$$$
\left.\leq 8^{p-1} c_{p} M^{p} k_{3}^{p}\left[\int_{0}^{t} e^{-2 a(t-s)}(E \| x(s-\sigma(s))) \|_{X}^{p}\right)^{(2 / p)} d s\right]^{(p / 2)}
$$

$$
\leq 8^{p-1} c_{p} M^{p} k_{3}^{p}\left[\int_{0}^{t_{1}} e^{-2 a(t-s)}(E \| x(s-\sigma(s))) \|_{X}^{p}\right)^{(2 / p)} d s
$$

$$
\begin{gathered}
\left.\left.+\int_{t_{1}}^{t} e^{-2 a(t-s)}(E \| x(s-\sigma(s))) \|_{X}^{p}\right)^{2 / p} d s\right]^{p / 2} \\
\left.\leq 8^{p-1} c_{p} M^{p} k_{3}^{p} \int_{0}^{t_{1}}\left(e^{-2 a(t-s)}(E \| x(s-\sigma(s))) \|_{X}^{p}\right)^{(2 / p)}\right)^{(p / 2)} d s \\
+8^{p-1} c_{p} M^{p} k_{3}^{p} \varepsilon(2 a)^{-p / 2}
\end{gathered}
$$

As $e^{-p a t} \rightarrow 0$ as $t \rightarrow \infty$ and by assumption on Theorem 3.1 , there exists a $t_{2}>t_{1}$, such that for any $t \geq t_{2}$, we have

$$
\begin{gathered}
\left.8^{p-1} c_{p} M^{p} k_{3}^{p} \int_{0}^{t_{1}}\left(e^{-2 a(t-s)}(E \| x(s-\sigma(s))) \|_{X}^{p}\right)^{(2 / p)}\right)^{(p / 2)} d s \\
\leq \varepsilon-8^{p-1} c_{p} M^{p} k_{3}^{p} \varepsilon(2 a)^{-p / 2} .
\end{gathered}
$$

From (18) and (19), for any $t \geq t_{2}$, we obtain

$$
8^{p-1} E\left\|\int_{0}^{t} S(t-s) f_{2}(s, x(s-\sigma(s))) d w(s)\right\|_{X}^{p}<\varepsilon .
$$

That is to say

$$
8^{p-1} E\left\|\int_{0}^{t} S(t-s) f_{2}(s, x(s-\sigma(s))) d u(s)\right\|_{X}^{p} \rightarrow 0 \text { as } t \rightarrow \infty
$$

Thus from (8)-(12) and (16), (17), (21), we can obtain $E\|(\Psi x)(t)\|_{x}^{p} \rightarrow 0$ as $t \rightarrow \infty$. Thus we conclude that $\Psi(H) \subset H$.

Next we prove that $\Psi$ is a contraction mapping .To see this, Let $x, y \in H$ and for $s \in[0, T]$, we obtain

$$
\sup _{s \in[0, T]} E\|(\Psi x)(t)-(\Psi y)(t)\|_{X}^{p}
$$

$$
\begin{aligned}
& \leq 5^{p-1} \sup _{s \in[0, T]} E\left\|\int_{0}^{t} C(t-s)\left(\begin{array}{c}
f_{0}(s, x(s-\delta(s))) \\
-f_{0}(s, y(s-\delta(s)))
\end{array}\right) d s\right\|_{X}^{p} \\
& +5^{p-1} \sup _{s \in[0, T]} E\left\|\int_{0}^{t} S(t-s)\left(\begin{array}{c}
f_{1}(s, x(s-\rho(s))) \\
-f_{1}(s, y(s-\rho(s)))
\end{array}\right) d s\right\|_{X}^{p}
\end{aligned}
$$




$$
\begin{aligned}
& +5^{p-1} \sup _{s \in[0, T]} E\left\|\int_{0}^{t} S(t-s)\left(\begin{array}{c}
f_{2}(s, x(s-\sigma(s))) \\
-f_{2}(s, y(s-\sigma(s)))
\end{array}\right) d \omega(s)\right\|_{X}^{p} \\
& +5^{p-1} \sup _{s \in[0, T]} E\left\|\sum_{0<t_{k}<t} C\left(t-t_{k}\right)\left(I_{k}\left(x\left(t_{k}\right)\right)-I_{k}\left(y\left(t_{k}\right)\right)\right)\right\|_{X}^{p} \\
& +5^{p-1} \sup _{s \in[0, T]} E\left\|\sum_{0<t_{k}<t} S\left(t-t_{k}\right)\left(\tilde{I}_{k}\left(x\left(t_{k}\right)\right)-\widetilde{I}_{k}\left(y\left(t_{k}\right)\right)\right)\right\|_{X}^{p} \\
& \leq 5^{p-1} M^{p}\left[k_{1}^{p} b^{-p}+k_{2}^{p} a^{-p}+k_{3}^{p} c_{p}(2 a)^{-p / 2}\right. \\
& \left.+e^{-p b T} E\left(\sum_{k=1}^{m}\left\|q_{k}\right\|_{X}^{p}\right)+e^{-p a T} E\left(\sum_{k=1}^{m}\left\|g_{k}\right\|_{X}^{p}\right)\right] \\
& \times \sup _{s \in[0, T]} E\|x(t)-y(t)\|_{X}^{p} \cdot
\end{aligned}
$$

Therefore, $\Psi$ is a contraction mapping and hence there exist an unique fixed point $x(\cdot)$ in $H$ which is the solution of the equations $\quad(1)-(3) \quad$ with $x_{0}(\cdot)=\varphi, x^{\prime}(0)=x_{1}$ and $E\|x(t)\|_{X}^{p} \rightarrow 0$ as $t \rightarrow \infty$. This completes the proof.

\section{Corollary 3.2:}

If the conditions (I) to (IV) hold, then the second- order neutral impulsive stochastic differential system (1) to (3) is mean square asymptotically stable if

$$
\begin{aligned}
& 5 M^{2}\left(k_{1}^{2} b^{-2}+k_{2}^{2} a^{-2}+k_{3}^{2}(2 a)^{-1}+\hat{L}+\hat{D}\right)<1 \\
& \text { where } \hat{L}=e^{-2 b T} E\left(\sum_{k=1}^{m}\left\|q_{k}\right\|_{X}^{2}\right) \\
& \hat{D}=e^{-2 b T} E\left(\sum_{k=1}^{m}\left\|g_{k}\right\|_{X}^{2}\right)
\end{aligned}
$$

\section{REFERENCES}

[1] Appleby, J. A. D., (2008), Fixed points, stability and harmless stochastic perturbations, preprint.

[2] Balasubramaniam, P., and Muthukumar, P., Approximate controllability of second-order stochastic distributed implicit functional differential systems with infinite delay, J. Optim. Theory Appl., 143 (2) (2009), 225-244.

[3] Caraballo, T., Asymptotic exponential stability of stochastic partial differential equations with delay, stochastics, 33 (1990), 27- 47.

[4] Caraballo, T., Real, J., Partial differential equations with delayed random perturbations: existence, uniqueness and stability of solutions, Stoch. Anal. Appl. 11 (1993), $497-$ 511.

[5] Caraballo, T., Real, J., Taniguchi, T. The exponential stability of neutral stochastic delay partial differential equations, Discrete and continuous Dynamical systems, series A, 18 (2-3) (2007), 295-313.

[6] Da Prato, G., Zabczyk, J., 1992. Stochastic Equations in infinite Dimensions (Cambridge University Press, Cambridge)

[7] Dauer, J.P., Mahmudov, N. I., Integral inequalities and mild solutions of semilinear neutral evolution equations, J. Math. Anal. Appl. 300 (1) (2004), 189-202.

[8] Fattorini, H.O., 1985. Second order linear Differential equations in Banach Spaces, North Holland Mathematics Studies series No. 108 (Elsevier Science, North Holland).

[9] Hale, J. K., Lunel. S. M. V., 1991. Introduction to Functional differential equations (Springer-Verlag, Berlin).

[10] Hu, L., Ren, Y., Existence results for impulsive neutral stochastic functional integro-differential equations with infinite delays, Acta Appl. Math. 111 (3) (2010), 303-317.

[11] Ichikawa, A., Stability of semilinear stochastic evolution equations, J. Math. Anal. Appl. 90 (1982), 12-24.

[12] Khas'minskii, R., 1980. Stochastic stability of differential equations, Sijthoff and noordhoff, Netherlands.

[13] Kolmanovskii, V. B and., Myshkis, A., 1992. Applied Theory of Functional Differential Equations (Kluwer Academic, Norwell, MA).

[14] Lei Zhang, Yongsheng Ding, Tong Wang, Liangjian Hu, and Kuangrong Hao, Controllability of second-order semilinear impulsive stochastic neutral functional evolution equations, Mathematical Problems in Engineering 748091 (2012), 1-13.

[15] Liu, K., Truman, A., A note on almost exponential stability for stochastic partial differential equations, Statist. Probab. Lett. 50 (2000), 273-278.

[16] Luo, J., Fixed points and exponential stability of mild solutions of stochastic partial differential equations with delays, J. Math. Anal. Appl. 342 (2008), 753-760.

[17] Luo, J., Stability of stochastic partial differential equations with infinite delays, J. Comput. Appl. Math. 222 (2008), 364-371.

[18] Luo, J., Exponential stability for stochastic neutral partial functional differential equations, J. Math. Anal. Appl. 355 (1) (2009), 414-425.

[19] Luo, J., Taniguchi, T., Fixed points and stability of stochastic neutral partial differential equations with 
International Journal of Computer Applications Technology and Research

Volume 2- Issue 4, 415 - 421, 2013

infinite delays, Stochastic Analysis and Applications, 27

(2) (2009), 1163-1173.

[20] Mahmudov, N. I., and Mckibben, M. A., Abstract second-order damped Mckean-Vlasov stochastic evolution equations, Stoch. Anal. Appl. 24 (2006), 303-328.

[21] Nieto, J.J., Rodriguez-Lopez, R., Boundary value problems for a class of impulsive functional equations, Comput. Math. Appl. 55 (2008), 2715-2731.

[22] Nieto, J.J., Rodriguez-Lopez, R., New comparison results for impulsive integro-differential equations and applications, J. Math. Anal. Appl. 328 (2007), 1343-1368.

[23] Ren, Y., Dandan, S., Second order neutral impulsive stochastic evolution equations with delays, J. Math. Phys. 50 (2009), 102709.

[24] Sakthivel, R., Ren, Y., Kim, H., Asymptotic stability of second-order neutral stochastic differential equations, J. Math. Phys. 51 (2010), 052701.

[25] Samoilenko, A.M., Perestyuk, N.A., 1995. Impulsive Differential Equations, World Scientific, Singapore.

[26] Taniguchi, T., Liu, K., A., Truman, Existence and uniqueness and asymptotic behavior of mild solution to stochastic functional differential equations in Hilbert spaces, J. Differential Equations 18 (2002), 72-91.

[27] Travis, C.C., Web, G.F., Cosine families and abstract nonlinear second order differential equations, Acta Math. Acad. Sci. Hung. 32 (1978), 75-96. 